\title{
Exploring the Role of Emotions and Moods in Decision Making: Study on the Use of Structured Decision Approach and Intuition
}

\author{
S. ZULFIQAR, A. ISLAM \\ Károly Ihrig Doctoral School of Management and Business, University of Debrecen, Debrecen, Hungary, \\ sehar.zulfiqar@econ.unideb.hu \\ Capital University of Science and Technology, Islamabad, Pakistan, amjadswaati@yahoo.com
}

Abstract. Emotions and moods can play a significant role in the decision making. The present paper contest this point by providing evidence on the two important approaches used in decision making that is structured decision approach and intuition. For this purpose data was gathered from 150 respondents in two different groups. Chi square test, independent sample t-test and logistic regression analysis were used to test the hypothesis. The results of the study reveal that individuals with positive and negative mood state vary significantly in their use of structured decision approach and intuition. The individuals with negative mood state are more likely to use all steps of structured decision approach as compared to the individuals in the positive mood state. The results of the study further reveal that the individual's emotional state significantly predicted the use of structured approach and intuition in decision making.

\section{Introduction}

Decision making is a complex rational process studied by numerous researchers. For long it was believed that since decision making is cognizant; so emotions and moods don't have any role to play in this process [1]. Modern economic theory almost overlooked the idea that emotions can or should play any role in the decision making process mainly due to the fact that emotions and moods have impaired history. Historically, this remained a major challenge for the psychologists to answer the queries like what exactly moods and emotions are, what is included in them and how to evaluate them. But now the situation is different with overwhelming evidence on the role of emotions and moods by and large all the current theories of decision making include the cognitive perspective. It is assumed that decisions are the result of some cost and benefit analysis and human feelings can affect this analysis [2]. The emerging field of Neuroeconomics in this regard provides the major evidence by arguing that there is a brain region called the anterior insula activated during the decision making process; this region of the brain is actually responsible for emotional processing and affects the process of constructing decisions [3]. 
Although in recent years; growing attention has been given to the role of human feelings in the decision making, but still the gap exists that how the individuals construct their decisions and how the human feelings can affect this process. The individuals usually construct their decisions by following two approaches either they follow the structured decision approach in which they generate, evaluate and carefully choose between the alternatives or make decisions based on their intuitions. There can be a possibility that emotions and moods can influence the individual's choice of these two alternate approaches for decision making i.e. intuition verses structured decision approach. This knowledge can have relevance for different areas, e.g. in the field of marketing this information that how different feelings affect the purchasing decisions of consumers, can help companies better plan their marketing schemes [4]. So this article is intended to explore the role of human feelings in the decision making by specifically highlighting how emotions and moods can affect the use of structured decision approach and exercise of intuition in the process of constructing decision. The article consists of two parts: first part provides the review of current literature that underpins the notion that emotions and moods have a significant part in the decision making and second part will provide the empirical evidence on the connection between emotions, moods and decision making by addressing the specific aspect of intuitive and structured decision approaches.

\section{Literature Review}

Emotions and moods are two distinct constructs, although literature provides clear differentiation, but often confused with one another. Emotions are specific temporary subjective feelings (e.g. Glad, sad, angry, etc.) generally caused by some identifiable source. Whereas moods on the other hand, are tend to be long lasting and weak, but don't have any clear beginning. Moreover moods are more diffused and can exist in the form of positive and negative moods [5]. Another distinction between the two is that moods are not task related, but emotions are task-sensitive [6].

Further emotions have three primary dimensions: 1) valence (pleasantness/unpleasantness of the experience), 2) arousal (activation generated by the experience), and 3) dominance (degree of control that one is able to exercise over the emotional experience induced [7] [8]. And these dimensions can be applied to explain the emotional differences of people and then how these differences connect to decision making. For example, people with positive valence tend to choose utilitarian choices as compare to the people having negative valance who tend to choose deontological choices.

According to researchers emotions cannot be separated from decision making, but actually they are part of the decision process and serves four functions: 1) information about pain and pleasure to build preference, 2) facilitates speedy choices because of deadlines, 3) to help concentrate on the important issues connected with decision problem, 4) to create assurance regarding morally and socially significant decisions [9].

In the recent years the most dominant framework regarding the debate on the role of emotions in decision making process is the somatic marker hypothesis, according to this hypothesis human behavior particularly decision making is guided by emotions. Somatic marker are feelings in the body that are associated with the emotions. In the decision making process individuals use cognitive or 
emotional processes to access the options available to them. In the process, an individual came across physical changes that are transmitted to the brain where they are translated into emotions that tell the individual something about the encountered stimulus. Damasio, termed these changes in the body and brain states as emotions. Emotions and corresponding body changes are called as somatic marker. When the somatic marker having negative outcome is perceived, it alarms the individual to avoid the behavior and similarly the somatic marker with positive outcome make the individual happy and encourage them to follow the outcome [3] [10]. Emotions linked to a particular incident can have a long term effect on the decision making. Normally people tend to remember their past experiences; the choices they made and their emotional reactions at particular places to particular incidents [5]. Later, in the long run these incidental emotions can affect the decisions making process. As they provide the basis for evaluation of a certain outcome in the future. These emotions help people transform the probabilities of profits or losses into respective weights and values. In this regard the Bayesian statistical models it can be said to explain that the alterations in belief (past probabilities) and proof (likelihood) can be a function of individual's mood states [11].

Another model that helps understand the complex social decision making process is Emotions as Social Information (EASI) it explains that how the social interaction affect the decision making by arguing that emotional states of the interdependent party can influence the selection of the decision maker in the ambiguous situation and furthermore the nature of the situation (cooperative verses competitive) effect this social interaction. Different emotional states can be experienced and expressed in the social decision making process and this EASI model categorizes them into four types 1) opportunity/affiliation (happiness and related emotions), 2) dominance/aggression (anger and related emotions), 3) supplication (sadness and related emotions), and 4) appeasement (guilt and related emotions) [12].

According to Leone et al [13], the effect of positive and negative emotions on decision making is asymmetric in nature. People in a negative emotional state require more reasoning as compare to the people in the positive emotional state to understand why and how they are experiencing the unpleasant feelings. They have more strategic orientation towards creating the decisions, e.g., people in a negative emotional state when decide to help others, they have strategic reasons which are opposite for individuals in positive mood states who are more mindless when deciding to help others.

Like emotions, moods also tend to affect the decision making process and there is considerable research evidence that supports this notion. These effects can be constructive or destructive and can influence the effectiveness of the decision making process in both positive and negative ways. E.g. people with positive mood state tend to remember more information and make less conservative judgments as compared to the people in the negative mood state [14].

There also exist a strong connection between moods and risky decisions. When people are in a good mood they tend to avoid risky decisions so to avoid messing up their positive mood. But in lower-risk situations, they are more willing to make risky decisions because the gain is more likely to happen which can help them maintain their good moods [15]. 
Another interesting aspect of human feelings and decisions is the use of intuition in the decision making process. It's worth noting that the synonyms of intuition are; gut feeling or gut instinct, that in itself show that there is a cognitive process active. So the intuitive judgments have affective component and it can be said that as they are affective in nature as they are away from rationality. Since rationality is caused by head and judgments are caused by the brain. Evidence suggests that managers are more likely to make intuitive judgments when they are experiencing a feeling of excitement and harmony. So it can be concluded that when people are in a good mood they tend to make more intuitive judgements as compare to the people who are experiencing negative feelings [16].

The similar evidence comes from the ultimatum game. In the ultimatum game the sum of money is to be split between the initiator and the respondent. The initiator floats the offer and the responder has to accept or decline the offer. If the responder rejects the offer the game will be lost and neither the initiator nor the responder will receive any money. Interestingly the rejection rate for the offer is less than $20 \%$ and usually high for humans comparing with those of computers. Because, the humans respond differently to the unfair offers. Here the possible justification to the different response is that offer less than $20 \%$ triggers negative feelings and negative emotional state leads to the rejection of the offer [17].

On the basis of the literature reviewed following hypothesis are generated:

Hypothesis 1: Emotion is a significant predictor of intuition in decision making.

Hypothesis 2: Emotion is a significant predictor of structured decision making approach.

Hypothesis 3: Individuals with negative mood are significantly different from the individuals with positive mood in their use of the structured decision approach

Hypothesis 4: Individuals with negative mood are significantly different from the individuals with positive mood in their use of intuition in making decision.

Hypothesis 5: Individuals with negative mood are more likely to use all steps of structured decision approach in making a decision as compared to the individuals with positive mood.

\section{Methodology}

\subsection{Participants}

The participants of the study were the students enrolled in the MBA program (Master of Business Administration) in the National university of Modern Languages, Islamabad, Pakistan. The 150 students from the final semester of the evening program were selected for the current survey. The rationality of choosing evening students were that they can be considered good proxies for the middle level managers as majority of the students were employed so they were having professional experience and sufficient knowledge about the decision making process. 


\subsection{Instrumentation and Measurement}

Moods and Emotions were the two independent variables, whereas decision making process was the dependent variable of the study. Moods and decision making process were evaluated utilizing the methodology of Elsbach \& Pamela [18]. The scale for moods measured the two conditions (a) positive and (b) negative moods. Whereas the scale for decision making measured the three important aspects of the decision making process (a). Use of all the steps of a structured decision making process, (b) use of steps in the accurate order and (c) use of intuition verses the result of a structured decision making process to arrive the conclusion. Emotions were measured by using the paper and pencil version of self-assessment manikins (SAMs) [19]. SAMs measure the emotional responses on three dimensions (a) valence (or pleasure), (b) arousal, and (c) dominance.

\subsection{Procedure}

Following the methodology of [20] all students were given the lecture on structured decision making process. Before the start of the lecture all students were given the self-assessment manikins (SAMs) and were asked to check the three frames in three rows that best identify their emotional status at that time. After the lecture students were split into two groups. One group was given the negative mood treatment by demanding them to solve the extremely difficult anagrams and the other group was given the positive mood treatment by giving them pack of candies. Subsequently, after the use of mood manipulators the students of both groups were required to fill the mood questionnaire in order to track their mood at that particular time.

In order to measure the decision making process the students of both groups were asked to solve the famous business case "The Carter Racing Case" [21]. The students were supposed to the reach the decision after reading the case that whether Carter should or shouldn't participate in the next race. For this task; students were given twenty minutes. After the completion of the task the students were given the decision making questionnaire that included questions regarding the use of structured verse unstructured approach to decision making. How accurately the respondents, followed the steps of the structured decision making process and in the end, what respondents preferred; the gut feeling or the outcomes of the structured decision making approach to arrive at the final decision.

\section{Results}

In order to test the hypothesis 1 and 2; logistic regression analysis was performed to predict the use of a structured approach and intuition in decision making by using emotion as a predictor.

\begin{tabular}{|l|l|l|l|}
\hline & B & Standard Error & Wald \\
\hline Emotion & -1.082 & .326 & $11.014^{* * *}$ \\
\hline
\end{tabular}

Statistical significance: ${ }^{*} \mathrm{p}<.05 ;{ }^{* *} \mathrm{p}<.01 ;{ }^{* * *} \mathrm{p}<.001$

Table 1: Logistic Regression Analysis Showing the Effect of Emotions in the Prediction of Structured Decision Approach 


\section{(Author's Calculation)}

Results show that test of the full model against a constant only model is found to be statistically significant, indicating that the emotion reliably distinguished between the use of structured approach or not in the decision making (chi square $=13.369, \mathrm{p}<.001$ with $\mathrm{df}=1$ ). Nagelkerke's R2 of .130 indicated a weak relationship between emotion and use or no use of a structured approach in decision making. Prediction success was overall 63.4\% (49.2\% for No and $75.7 \%$ for Yes). The Wald criterion demonstrated that emotions made a significant contribution to the prediction of the dependent variable ( $\mathrm{p}=.001)$. Exp (B) value indicates that when emotion is raised by one unit the odds ratio is 0.339 times as large. Specifically, when emotion is raised by 1 unit, individuals become 0.339 less likely to use a structured approach in decision making.

A logistic regression analysis was performed to predict the use of intuition in decision making by using emotion as a predictor and it shows the following result.

\begin{tabular}{|l|l|l|l|}
\hline & B & Standard Error & Wald \\
\hline Emotion & .949 & .313 & $9.176^{* * *}$ \\
\hline
\end{tabular}

Statistical significance: ${ }^{*} \mathrm{p}<.05 ;{ }^{* *} \mathrm{p}<.01 ;{ }^{* * *} \mathrm{p}<.001$

Table 2: Logistic Regression Analysis Showing the Effect of Emotions in the Prediction of Intuition

\section{(Author's Calculation)}

A test of the full model against a constant only model was found to be statistically significant, indicating that the predictor reliably distinguished between the use of intuition or not in the decision making (chi square $=10.727, \mathrm{p}<.001$ with $\mathrm{df}=1$ ). Nagelkerke's R2 of .150 indicated a weak relationship between emotion and use or not use of intuition in decision making. Prediction success overall was $61.8 \%$ ( $75 \%$ for use of intuition and $46.7 \%$ for no use of intuition). The Wald criterion demonstrated that emotions made a significant contribution to the prediction of the dependent variable ( $\mathrm{p}=.002)$. Exp (B) value indicates that when emotion is raised by one unit the odds ratio is 2.582 times as large. Specifically, when emotion is raised by 1 unit, individuals become 2.58 more likely to use intuition in decision making.

Table 3 and 4 shows the chi square statistics. The results show that hypothesis 3 is accepted, as there is a significant association between the mood state and use of the structured decision approach, $\chi^{2}(1)$ $=21.165, p=.000$. The individuals with negative mood state were more likely to use a structured approach in making decisions than the individuals with positive mood state.

\begin{tabular}{|c|c|c|c|}
\hline & \multicolumn{3}{|c|}{ Use Of Structured Decision Approach } \\
\hline Mood State & Yes & No & Total \\
\hline Positive & $29.0 \%(18)$ & $69 \%(49)$ & $50.4 \%(67)$ \\
\hline Negative & $71 \%(44)$ & $31.0 \%(22)$ & $49.6 \%(66)$ \\
\hline Total & $100 \%(62)$ & $100 \%(71)$ & 133 \\
\hline$\chi^{\mathbf{2}} \mathbf{1} \mathbf{= 2 1 . 1 6 5 * *}$ & & & \\
\hline
\end{tabular}


Statistical significance: ${ }^{*} \mathrm{p}<.05 ;{ }^{* *} \mathrm{p}<.01 ;{ }^{* * *} \mathrm{p}<.001$

Table 3: Chi-Square Statistics for Moods and Use Structured Decision Approach

(Author's Calculation)

Similarly hypothesis 4 is also accepted, as there was a significant difference found between the observed and expected frequency of individuals with negative and positive mood state in their use of intuition in decision making $\chi^{2}(1)=21.121, p=.000$.

\begin{tabular}{|c|c|c|c|}
\hline & \multicolumn{3}{|c|}{ Use Of Intuition } \\
\hline Mood State & Yes & No & Total \\
\hline Positive & $69.6 \%(48)$ & $29.7 \%(19)$ & $50.4 \%(67)$ \\
\hline Negative & $30.4 \%(21)$ & $70.3 \%(45)$ & $49.6 \%(66)$ \\
\hline Total & $100 \%(69)$ & $100 \%(64)$ & 133 \\
\hline$\chi^{\mathbf{2}}$ (1) =21. 121*** & & & \\
\hline
\end{tabular}

Statistical significance: ${ }^{*} \mathrm{p}<.05 ;{ }^{* *} \mathrm{p}<.01 ;{ }^{* * *} \mathrm{p}<.001$

Table 4: Chi-Square Statistics for Moods and Use of Intuition

(Author's Calculation)

\begin{tabular}{|l|l|l|l|l|l|}
\hline & \multicolumn{2}{|l|}{$\begin{array}{l}\text { Negative Mood } \\
(\mathbf{N = 6 6 )}\end{array}$} & $\begin{array}{l}\text { Positive Mood } \\
(\mathbf{N = 6 7 )}\end{array}$ & \\
\hline Scales & $M$ & $S D$ & $M$ & $S D$ & $T$ \\
\hline Steps of Structured Decision Approach & 3.13 & 1.25 & 2.28 & 1.19 & $-4.01^{* * *}$ \\
\hline
\end{tabular}

Table 5: Mean, Standard Deviation and $t$ values for positive and negative mood states on the relative use of structured decision making steps $(N=133)$

(Author's Calculation)

Hypothesis 5 stated that individuals with negative mood state will use more steps of the structured decision approach as compared to the individuals with positive mood state. An independent samples ttest was used to find if there was a significant difference between the individuals in positive and negative mood states in their use of steps of structured decision approach. This analysis found a significant difference between the two groups $t(131)=-4.01, p<.000)$ in their use of steps. Individuals with negative mood state significantly scored higher on the use of steps of structured decision approach as compared to the individuals with positive mood state. Individuals with negative mood $(M$ $=3.13$ ) significantly higher on their use of a structured approach that they were more likely to use on average 3 steps of structured decision approach face than the individuals with positive mood state $(M$ $=2.28$ ) who reported to use on average 2 steps of structured decision approach. 


\section{Discussion and Conclusion}

The major findings of this study suggest that individuals in negative mood state are more likely to use structured decision approach in decision making as compared to the individuals in the positive mood state who rely more on intuition while constructing decisions. Moreover, the results establish that people in negative mood state are inclined to use more steps of structured approach in decision making than the individuals in a positive mood state. Another finding of the survey is that emotions significantly predicted the role of structured decision approach and intuition in decision making. These findings are in correspondence with the findings of previous studies [16] [18]. The literature supports various reasons, e.g. the temporal proximity of decision tends to increase the reliance of people on their affective system. The affective feelings have a more intensive influence on decisions for which the outcome is in the near future as compared to the one for which the reward is at a remote time. The proximity to the present outcome has a tendency to not only increase the intensity of the feelings, but also increase the reliance on these feelings in making judgments [22].

Moreover, both positive and negative emotions have motivational influences, but these influences can take different paths and different directions. Negative emotions adopt a relatively more complex path during the evaluative stage of decision making. Negative emotional states also push the individuals to adapt the evaluative paths in such a way so they can reduce the risk of negative outcomes [13].

Lastly, it can be said that emotions and moods affect the decision making process, but the use of intuition in the decision making process is influenced by numerous factors, such as the domain knowledge, learning and problem structure. For example, it can be argued that the problem exists in the form of a continuum, one side of the continuum is structured problems and the other side is having unstructured problems. When the problem is unstructured the individuals are more likely to rely on the use of intuition [16]. The research on the role of emotions and moods need to be expanded to develop the clear understanding of how human feelings can affect the complex process of constructing decisions.

\section{References}

[1] Peters, E., Va"stfja“ll, D., Ga"rling, T., \& Slovic, P. (2006). Affect and decision making: A "hot" topic.Journal of Behavioral Decision Making,19, 79-85

[2] Bechara A., Damasio H., Damasio A. R. (2000). Emotion, decision making and the orbitofrontal cortex. Cerebral Cortex, 10(3), 295-307.

[3] Bechara A., Damasio A. R. (2005). The somatic marker hypothesis: A neural theory of economic decision. Games and Economic Behavior, 52(2), 336-372.

[4] San Yap, C. W., \& Yazdanifard, R. (2014). How consumer decision making process differ from youngster to older consumer generation. Journal of Research in Marketing, 2(2), 151-156. 
[5] Andrade, E. B., \& Ariely, D. (2009). The enduring impact of transient emotions on decision making. Organizational Behavior and Human Decision Processes, 109(1), 1-8.

[6] Chung, J. O., Cohen, J. R., \& Monroe, G. S. (2008). The effect of moods on auditors' inventory valuation decisions. Auditing: A Journal of Practice \& Theory, 27(2), 137-159.

[7] Greenwald, M. Ks., Cook, E. W., \& Lang, P. J. 1989!. Affective judgment and psychophysiological response: Dimensional covariation in the evaluation of pictorial stimuli. Journal of Psychophysiology, 3, 51-64

[8] Lang, P. J., Greenwald, M. K., Bradley, M. M., \& Hamm, A. O. 1993!. Looking at pictures: Affective, facial, visceral, and behavioral reactions. Psychophysiology, 30, 261-273.

[9] Pfister, H. R., \& Böhm, G. (2008). The multiplicity of emotions: A framework of emotional functions in decision making. Judgment and decision making, 3(1), 5.

[10] Bosse, T., Hoogendoorn, M., Klein, M. C., Treur, J., Van Der Wal, C. N., \& Van Wissen, A. (2013). Modelling collective decision making in groups and crowds: Integrating social contagion and interacting emotions, beliefs and intentions. Autonomous Agents and MultiAgent Systems, 1-33.

[11] Paulus, M. P., \& Angela, J. Y. (2012). Emotion and decision-making: affect-driven belief systems in anxiety and depression. Trends in cognitive sciences, 16(9), 476-483.

[12] Van Kleef, G. A., De Dreu, C. K., \& Manstead, A. S. (2004). The interpersonal effects of anger and happiness in negotiations. Journal of personality and social psychology, 86(1), 57-76.

[13] Pascual-Leone, A., Amedi, A., Fregni, F., \&Merabet, L. B. (2005). The plastic human brain cortex. Annu. Rev. Neurosci., 28, 377-401.

[14] San Yap, C. W., \& Yazdanifard, R. (2014). How consumer decision making process differ from youngster to older consumer generation. Journal of Research in Marketing, 2(2), 151156.

[15] Hockey, G. R. J., John Maule, A., Clough, P. J., \&Bdzola, L. (2000). Effects of negative mood states on risk in everyday decision making. Cognition \& Emotion, 14(6), 823-855.

[16] Dane, E., \& Pratt, M. G. (2007). Exploring intuition and its role in managerial decision making. Academy of management review, 32(1), 33-54.

[17] Van'tWout, M., Kahn, R. S., Sanfey, A. G., \& Aleman, A. (2006). Affective state and decisionmaking in the ultimatum game. Experimental brain research, 169(4), 564-568.

[18] Elsbach, K. D., \& Barr, P. S. (1999). The effects of mood on individuals' use of structured decision protocols. Organization Science, 10(2), 181-198.

[19] Bradley, M. M., \& Lang, P. J. (1994). Measuring emotion: the self-assessment manikin and the semantic differential. Journal of behavior therapy and experimental psychiatry, 25(1), 49-59. 
[20] Elsbach, K. D., \& Barr, P. S. (1999). The effects of mood on individuals' use of structured decision protocols. Organization Science, 10(2), 181-198.

[21] Brittain, J. S. B. Sitkin. 1989. Facts, figures, and organizational decisions: Carter Racing and quantitative analysis in the organizational behavior classroom. Organ. Behavior Teaching Rev, 14(1), 62-81.

[22] Chang, H.H. and Pham, M.T., (2013). Affect as a decision-making system of the present. Journal of Consumer Research, 40(1), pp.42-63. 